\title{
On the Total Energy of Two-Electron Atoms *
}

\author{
B. Fricke \\ Universität Kassel, Institut für Theoretische Physik, Kassel, \\ Federal Republic of Germany \\ K. Rashid \\ Pakistan Institute of Nuclear Sciences and Technology, P.O. Nilore, and \\ Center of Basic Sciences, University Grants Commission, Islamabad, Pakistan \\ Received February 20, 1985
}

Using the Multi-Configuration Dirac-Fock (MCDF) method we calculate with 9 configuration state functions the correlation energy as well as the total energy of the lowest $J=0$ ground state of all two-electron systems from $\mathrm{H}^{-}$to Thorium $(Z=90)$. A comparison with experimental data, which are available only in the low $Z$ region, shows a very good agreement.

The total energy $E$ is the most important physical quantity which describes a quantum mechanical system. It appears directly in the stationary Schrödinger- or Dirac-equation, respectively. Thus, it is permissible to state: If the theory can describe this quantity with high accuracy, then the system can be said to be understood.

As the electromagnetic interaction is known very well, atomic systems with a small number of electrons are the standard examples of so-called simple systems. Nevertheless, it is well known that even the hydrogen atom has its complications, first because of the Lamb-shift, which contains the QED contributions, vacuum fluctuation, and vacuum polarisation, and secondly because of the influence of the extended nuclear charge together with its influence on the QED effects. A large number of papers have appeared on this subject, calculating the QED contributions to the best possible order $[1,2]$. Just recently, a summary was published by Mohr [3], where all contributions for one-electron systems are given for all elements $Z \leqq 40$, Johnson and Soff [4] extended this list up to $Z=110$. These activities coincide with the revived experimental interest of measuring the Lambshift [5] in one-electron systems with ever increasing $Z$.

Apart from one-electron systems it is also possible to measure and calculate one-hole systems. Photo-

\footnotetext{
* Dedicated to Prof. Dr. A. Steudel on the occasion of his 60 th birthday
}

electron spectroscopy, for instance, is a well known tool for this [6]. The interest in such systems is due to the fact that experiments for one-hole systems are much easier than Lamb-shift experiments on oneelectron systems. On the other hand, one-hole systems are theoretically much more complicated. First of all, the energy of the Coulomb interaction between the electrons from full Dirac-Fock calculations between the neutral and ionized states has to be calculated. Additionally, the magnetic interaction and retardation for all the electrons has to be added. Although this appeared at first to be an unattainable challenge, Desiderio et al. [7], and later Fricke et al. [8], succeeded in obtaining nearly perfect agreement of experiment and theory of inner shell transitions within the experimental error bars at that time. Because inner shell transitions in heavy electronic systems can now be measured with an ever increasing accuracy, Deslattes [9] drew the attention to a comparison of the experimentally measured $K_{\alpha}$ and $K_{\beta}$ lines with theoretical ones. At present a difference of a few $\mathrm{eV}$, only, remains to be explained. Deslattes [10] concluded that this difference is probably due to correlation not calculated for very heavy atoms so far.

To obtain an initial starting point for calculating (part of) the correlation energy as part of the total energy, even for high $Z$ elements, we performed Multi-Configuration Dirac-Fock (MCDF) calculations for the ground state $1 s^{2}$ of all two-electron systems for all $Z$. The MCDF-method has been ex- 
Table 1. Correlation energy in $\mathrm{eV}$ of the lowest $J=0$ ground state configuration of two-electron systems from $\mathrm{H}^{-}$to $\mathrm{Th}^{88+}$ as well as the weights of the configurations

\begin{tabular}{|c|c|c|c|c|c|c|c|}
\hline \multirow[t]{2}{*}{ Element } & \multirow[t]{2}{*}{$E_{c}(\mathrm{eV})$} & \multicolumn{6}{|c|}{ Weights of the Configurations } \\
\hline & & $1 s^{2}$ & $2 s^{2}$ & $2 p_{1 / 2}^{2}+2 p_{3 / 2}^{2}$ & $3 s^{2}$ & $3 p_{1 / 2}^{2}+3 p_{3 / 2}^{2}$ & $3 d_{3 / 2}^{2}+3 d_{5 / 2}^{2}$ \\
\hline${ }_{1} \mathrm{H}^{-}$ & 1.06 & 0.945157 & 0.041578 & 0.012583 & $1.51(-4)$ & $2.11(-4)$ & $3.18(-4)$ \\
\hline${ }_{2} \mathrm{He}$ & 1.04 & 0.991993 & $3.823(-3)$ & $3.884(-3)$ & $5.1 \quad(-5)$ & $1.02(-4)$ & $1.46(-4)$ \\
\hline${ }_{3} \mathrm{Li}^{+}$ & 1.12 & 0.996768 & $1.36(-3)$ & $1.729(-3)$ & $2.1 \quad(-5)$ & $4.8 \quad(-5)$ & $7.3 \quad(-5)$ \\
\hline${ }_{4} \mathrm{Be}^{2+}$ & 1.14 & 0.998249 & $6.95 \quad(-4)$ & $9.72(-4)$ & $1.2 \quad(-5)$ & $2.9 \quad(-5)$ & $4.3 \quad(-5)$ \\
\hline${ }_{5} \mathrm{~B}^{3+}$ & 1.14 & 0.998915 & $4.17 \quad(-4)$ & $6.15 \quad(-4)$ & $7.2(-6)$ & $1.8(-5)$ & $2.8 \quad(-5)$ \\
\hline${ }_{6} \mathrm{C}^{4+}$ & 1.15 & 0.999256 & $2.80 \quad(-4)$ & $4.27 \quad(-4)$ & $5.0 \quad(-6)$ & $1.3(-5)$ & $2.0 \quad(-5)$ \\
\hline${ }_{7} \mathrm{~N}^{5+}$ & 1.15 & 0.999458 & $2.00 \quad(-4)$ & $3.13 \quad(-4)$ & $3.7(-6)$ & $9.4(-6)$ & $1.5(-5)$ \\
\hline${ }_{8} \mathrm{O}^{6+}$ & 1.15 & 0.999588 & $1.51(-4)$ & $2.40 \quad(-4)$ & $2.9(-6)$ & $7.3(-6)$ & $1.1 \quad(-5)$ \\
\hline${ }_{9} \mathrm{~F}^{7+}$ & 1.15 & 0.999666 & $1.18(-4)$ & $1.89 \quad(-4)$ & $2.3(-6)$ & $5.8 \quad(-6)$ & $9.0 \quad(-6)$ \\
\hline${ }_{10} \mathrm{Ne}^{8+}$ & 1.15 & 0.999738 & $9.4 \quad(-5)$ & $1.53 \quad(-4)$ & $1.9(-6)$ & $4.8 \quad(-6)$ & $7.4(-6)$ \\
\hline${ }_{12} \mathrm{Mg}^{10+}$ & 1.16 & 0.999820 & $(-5)$ & $1.06(-4)$ & $1.3(-6)$ & $3.3(-6)$ & $5.1(-6)$ \\
\hline${ }_{14} \mathrm{Si}^{12+}$ & 1.16 & 0.999868 & $(-5)$ & $7.7 \quad(-5)$ & $9.2 \quad(-7)$ & $2.4(-6)$ & $3.8 \quad(-6)$ \\
\hline${ }_{16}^{14} \mathrm{~S}^{14+}$ & 1.16 & 0.999900 & $(-5)$ & $(-5)$ & $7.2 \quad(-7)$ & $1.8(-6)$ & $2.9 \quad(-6)$ \\
\hline${ }_{18} \mathrm{Ar}^{16+}$ & 1.16 & 0.999921 & $(-5)$ & $(-5)$ & $5.7 \quad(-7)$ & $1.5(-6)$ & $2.3(-6)$ \\
\hline${ }_{20}^{10} \mathrm{Ca}^{18+}$ & 1.16 & 0.999936 & $(-5)$ & $(-5)$ & $4.7 \quad(-7)$ & $1.2(-6)$ & $1.9(-6)$ \\
\hline${ }_{25} \mathrm{Mn}^{23+}$ & 1.16 & 0.999958 & $(-5)$ & $(-5)$ & $3.1 \quad(-7)$ & $8.0 \quad(-7)$ & $1.2(-6)$ \\
\hline${ }_{30} \mathrm{Zn}^{28+}$ & 1.16 & 0.999971 & $(-5)$ & $(-5)$ & $2.3(-7)$ & $5.6(-7)$ & $8.3(-7)$ \\
\hline${ }_{40} \mathrm{Zr}^{38+}$ & 1.17 & 0.999984 & $(-6)$ & $(-6)$ & $1.4(-7)$ & $3.3(-7)$ & $4.5 \quad(-7)$ \\
\hline${ }_{50} \mathrm{Sn}^{48+}$ & 1.19 & 0.999989 & $(-6)$ & $(-6)$ & $9.6 \quad(-8)$ & $2.2(-7)$ & $3.0 \quad(-7)$ \\
\hline${ }_{60} \mathrm{Nd}^{58+}$ & 1.19 & 0.999993 & $(-6)$ & $(-6)$ & $7.4 \quad(-8)$ & $1.6 \quad(-7)$ & $2.1 \quad(-7)$ \\
\hline${ }_{70} \mathrm{Yb}^{68+}$ & 1.19 & 0.999994 & $(-6)$ & $(-6)$ & $6.1(-8)$ & $1.2(-7)$ & $1.5(-7)$ \\
\hline${ }_{80} \mathrm{Hg}^{78+}$ & 1.19 & 0.999996 & $(-6)$ & $(-6)$ & $5.4(-8)$ & $1.0 \quad(-7)$ & $1.1(-7)$ \\
\hline${ }_{90}^{0} \mathrm{Th}^{88+}$ & 1.21 & 0.999997 & $(-6)$ & $(-6)$ & $5.0 \quad(-8)$ & $8 \quad(-8)$ & $9 \quad(-8)$ \\
\hline
\end{tabular}

plained in various publications [11]. With this method the wave function $|\Psi\rangle$ is expanded in so-called configuration state functions $\left|\Psi_{\mathrm{CSF}}\right\rangle$, which are eigenfunctions to $J^{2}$

$|\Psi\rangle=\sum_{i} c_{i}\left|\Psi_{\mathrm{CSF}}\right\rangle$

The big problem within the actual calculation is the choice of the configurations to be included, and the convergence of the runs. In the case of $1 \mathrm{~s}^{2}(J=0)$, we chose to include the following nine configuration state functions: $1 s^{2}, 2 s^{2}, 2 p_{1 / 2}^{2}, 2 p_{3 / 2}^{2}, 3 s^{2}, 3 p_{1 / 2}^{2}$, $3 p_{3 / 2}^{2}, 3 d_{3 / 2}^{2}$, and $3 d_{5 / 2}^{2}$.

Table 1 lists the correlation energy $E_{c}$ for these twoelectron systems calculated with this procedure for a large number of elements through the whole Periodic System of Elements. Although the weights (i.e. $\left|c_{i}\right|^{2}$ of Eq. (1)) of the above mentioned nine configuration state functions within the $J=0$ ground state configuration, also listed in Table 1, change significantly with $Z$, the absolute contribution in energy is more or less constant over the entire range of $Z$ from negative Hydrogen to Thorium $(Z=90)$. For the extreme case of $\mathrm{H}^{-}$the $2 s^{2}$, and $2 p^{2}$ configurations together contribute more than $5 \%$, whereas for $\mathrm{Th}^{88}+$ their contribution is just $3.5 \mathrm{ppm}$.

For the low $Z$ elements the correlation energy is very well known from either very sophisticated pair- correlation [12] or CI [13] approaches, non-relativistic Multi-Configuration calculations [14], the Random-Phase approximation [15], or the double $Z^{-1}$ and $Z \alpha$ series expansion [16]. All these methods show that the inclusion of the lower $s^{2}$ and $p^{2}$ configurations already allow for about $90 \%$ of the correlation. Bunge's [13] result for $\mathrm{He}$, e.g. is $1.14 \mathrm{eV}$, which has to be compared with 1.04 from this MCDF method. The relative weights of the configurations with $n=2$ compared to those whith $n=3$, remain more or less constant. This indicates that the missing part of the correlation also remains about the same in absolute value. Nevertheless, we can expect that our values are a good first guess for the correlation of the $1 s^{2}$ two-electron ground state cases for' larger $Z$ elements.

Table 2 compares the theoretical total energy of such two-electron systems with the experimental total energies. The figures given here are results of the Multi-Configuration Dirac-Fock calculations including the effect of the extended nucleus in the second column, and the QED- and Breit-contributions in the third and fourth column. These figures show the well known fact that these contributions increase drastically with higher $Z$. A comparison with experimental total energies [17] $E_{\text {tot }}^{\exp }$ (which are the sum of the last two ionisation energies) up to $\mathrm{Mn}$ leads to very good agreement which in most cases is be- 
Table 2. Total energies of two-electron systems in their $J=0$ ground states from MCDF calculations including QED and Breit interaction plus a comparison with experimental data. All values are given in $\mathrm{eV}$

\begin{tabular}{|c|c|c|c|c|c|c|}
\hline Element & $\begin{array}{l}\text { MCDF (ext. } \\
\text { nucleus) }\end{array}$ & QED & Breit & $E_{\text {tot }}^{\mathrm{th}}$ & $E_{\mathrm{tol}}^{\exp }$ & $\Delta E$ \\
\hline${ }_{1} \mathrm{H}^{-}$ & 14.34 & 0.00 & 0.00 & 14.34 & 14.49 & 0.15 \\
\hline${ }_{2}^{1} \mathrm{He}$ & 78.97 & 0.00 & $-\quad 0.00$ & 78.97 & 79.00 & 0.03 \\
\hline${ }_{3}^{2} \mathrm{Li}^{+}$ & 198.06 & 0.00 & $-\quad 0.01$ & 198.05 & 198.09 & 0.04 \\
\hline${ }_{4} \mathrm{Be}^{2+}$ & 371.59 & 0.00 & $-\quad 0.02$ & 371.57 & 371.61 & 0.04 \\
\hline${ }_{5}^{4} B^{3+}$ & 599.61 & $-\quad 0.01$ & $-\quad 0.04$ & 599.56 & 599.58 & 0.02 \\
\hline${ }_{6} \mathrm{C}^{4+}$ & 882.15 & $-\quad 0.03$ & $-\quad 0.07$ & 882.05 & 882.06 & 0.01 \\
\hline${ }_{7}^{6} \mathrm{~N}^{5+}$ & $1,219.26$ & $-\quad 0.07$ & $-\quad 0.11$ & $1,219.08$ & $1,219.09$ & 0.01 \\
\hline${ }_{8} \mathrm{O}^{6+}$ & $1,610.99$ & $-\quad 0.11$ & $-\quad 0.16$ & $1,610.71$ & $1,610.70$ & -0.01 \\
\hline${ }_{9}^{0} \mathrm{~F}^{7+}$ & $2,057.40$ & $-\quad 0.18$ & $-\quad 0.24$ & $2,056.99$ & $2,056.96$ & -0.03 \\
\hline${ }_{10}^{3} \mathrm{Ne}^{8+}$ & $2,558.58$ & $-\quad 0.26$ & $-\quad 0.33$ & $2,557.99$ & $2,557.96$ & -0.03 \\
\hline${ }_{12} \mathrm{Mg}^{10+}$ & $3,725.55$ & $-\quad 0.51$ & $-\quad 0.58$ & $3,724.46$ & $3,724.41$ & -0.05 \\
\hline${ }_{14} \mathrm{Si}^{12+}$ & $5,112.65$ & $-\quad 0.90$ & $-\quad 0.93$ & $5,110.82$ & $5,110.71$ & -0.11 \\
\hline${ }_{16}^{14} S^{14+}$ & $6,720.80$ & $-\quad 1.45$ & $-\quad 1.40$ & $6,717.95$ & $6,717.94$ & -0.01 \\
\hline${ }_{18} \mathrm{Ar}^{16+}$ & $8,551.06$ & $-\quad 2.17$ & $-\quad 2.01$ & $8,546.87$ & $8,546.89$ & 0.02 \\
\hline${ }_{20} \mathrm{Ca}^{18+}$ & $10,604.62$ & $-\quad 3.05$ & $-\quad 2.78$ & $10,598.79$ & $10,598.75$ & -0.04 \\
\hline${ }_{25} \mathrm{Mn}^{23+}$ & $16,724.94$ & $-\quad 6.42$ & $-\quad 5.50$ & $16,713.02$ & $16,713.29$ & 0.27 \\
\hline${ }_{30} \mathrm{Zn}^{28+}$ & $24,275.74$ & -12.08 & $-\quad 9.61$ & $24,254.05$ & & \\
\hline${ }_{35} \mathrm{Br}^{33+}$ & $33,290.06$ & -20.12 & -15.42 & $33,254.52$ & & \\
\hline${ }_{40} \mathrm{Zr}^{38+}$ & $43,808.30$ & -31.56 & -23.24 & $43,753.51$ & & \\
\hline${ }_{45} \mathrm{Rh}^{43+}$ & $55,879.55$ & -46.49 & -33.41 & $55,799.64$ & & \\
\hline${ }_{50} \mathrm{Sn}^{48+}$ & $69,562.65$ & -66.08 & -46.31 & $69,450.25$ & & \\
\hline${ }_{55} \mathrm{Cs}^{53+}$ & $84,927.88$ & -90.43 & -62.33 & $84,775.12$ & & \\
\hline${ }_{60} \mathrm{Nd}^{58+}$ & $102,059.09$ & -120.75 & -81.90 & $101,856.44$ & & \\
\hline${ }_{65} \mathrm{~Tb}^{63+}$ & $121,054.75$ & -157.38 & -105.50 & $120,791.87$ & & \\
\hline${ }_{70} \mathrm{Yb}^{69+}$ & $142,030.56$ & -201.47 & -133.65 & $141,695.44$ & & \\
\hline${ }_{75} \mathrm{Re}^{78+}$ & $165,144.12$ & -254.24 & -167.01 & $164,722.87$ & & \\
\hline${ }_{80} \mathrm{Hg}^{78+}$ & $190,552.02$ & -316.74 & -206.22 & $190,029.06$ & & \\
\hline${ }_{85} \mathrm{At}^{83+}$ & $218,464.94$ & -390.13 & -252.12 & $217,822.69$ & & \\
\hline${ }_{90} \mathrm{Th}^{88+}$ & $249,144.52$ & -475.97 & -305.68 & $248,362.87$ & & \\
\hline
\end{tabular}

low $0.1 \mathrm{eV}$. This shows that the correlation energy of more than $1 \mathrm{eV}$ is a necessary part in order to obtain such a good comparison. Due to the inaccuracy of the correlation part we consistently present all values to the second decimal point, only. The highest $Z$ system where a comparison with experiment was possible can be found in the paper by Briand et al. [18], where the various contributions to several $\mathrm{X}$-ray transitions in two-electron $\mathrm{Fe}$ are discussed in great detail.

Summarizing we can say that two-electron systems even for higher $Z$ - can be understood with high accuracy, although a large number of theoretical problems are still unsolved, e.g. the influence of the QED contributions as well as the Breit term within the selfconsistent field process or the correlation effect of the Breit operator. Of course, the influence of such effects will probably only show-up for very large $Z$. On the other hand, the influence of the extended nucleus will bring in a large amount of uncertainties as well. In order to further improve the comparison, experimental data for even higher $Z$ elements are absolutely necessary.
Nevertheless, the values presented here are expected to be of high accuracy already.

The authors thank Dr. J.-P. Desclaux for interesting discussions and GSI for several hours of computing tims. The financial support from KfK and PAEC under the Pakistan-German Scientific Cooperation is greatly acknowledged.

\section{References}

1. A summary with all important references can be found in: Present status and aims of quantum electrodynamics. Lecture Notes in Physics, Gräff, G., Klempt, E., Werth, G. (eds.), Vol. 143. Berlin, Heidelberg, New York: Springer-Verlag 1980

2. Similar information can be obtained from the articles in: Relativistic effects in atoms, molecules and solids. NATO ASI Series. Malli, G.L. (ed.), Vol. 87. New York, London: Plenum Press 1983

3. Mohr, P.J.: At. Data. Nucl. Data Tables 29, 452 (1982)

4. Johnson, W.R., Soff, G.: (to be published)

5. Briand, J.P., Tavernier, M., Indelicato, P., Marrus, R., Gould, H.: Phys. Rev. Lett. 50, 832 (1983); Phys. Rev. A 28, 1413 (1983);

Briand, J.P., Indelicato, P., Tavernier, M., Gorceix, O., Liesen, D., Beyer, H.F., Liu, B., Warczak, A., Desclaux, J.P.: Z. Phys. A - Atoms and Nuclei 318, 1 (1984) 
6. A good example for a very heavy element is:

Porter, F.T., Freedman, M.S.: Phys. Rev. Lett 27, 293 (1971)

7. Desiderio, A.M., Johnson, W.R.: Phys. Rev. A 3, 1267 (1971)

8. Fricke, B., Desclaux, J.P., Waber, J.T.: Phys. Rev. Lett. 28, 714 (1972)

9. Deslattes, R.D., Kessler, E.G., Jacobs, L., Schmitz, W.: Phys. Lett. $71 \mathrm{~A}, 411$ (1979)

10. Deslattes, R.D.: Private communication

11. A few of the many examples are:

Desclaux, J.P.: Comput. Phys. Commun. 9, 31 (1975);

Desclaux, J.P.: p. 115 of Ref. [2];

Fricke, B.: Phys. Scr. T 8, 129 (1984)

12. A summary is given in the book:

Lindgren, I., Morrison, J.: Atomic many-body theory. In: Springer Series in Chemical Physics. Vol. 13. Berlin, Heidelberg, New York: Springer-Verlag 1982

13. e.g. Bunge, C.F.: Theor. Chim. Acta 16, $126(1970)$

14. e.g. Froese-Fischer, C.: J. Comput. Phys. 13, 502 (1972)

15. e.g., Johnson, W.R., Liu, C.D.: Phys. Rev. A 14, 565 (1976)

16. e.g., Safronova, U.I.: Phys. Scr. 23, 241 (1981)

17. Bashkin, S., Stoner, J.O.: Atomic energy levels and grotian diagrams. Amsterdam: North Holland 1975
18. Briand, J.P., Tavernier, M., Marrus, R., Desclaux, J.P.: Phys. Rev. A 29, 3143 (1984)

B. Fricke

Fachbereich 18

Theoretische Physik

Gesamthochschule Kassel

Postfach 101380

D-3500 Kassel-Oberzwehren

Federal Republic of Germany

\section{K. Rashid}

Pakistan Institute of Nuclear Sciences and Technology

and

Center of Basic Sciences

University Grants Commision

P.O. Nilore

Islamabad

Pakistan 